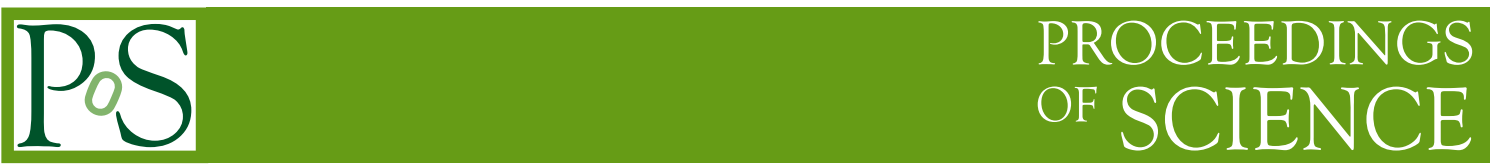

\title{
Introduction of bosonic fields into causal set theory
}

\author{
Roman Sverdlov* \\ Physics Department, University of Michigan \\ 450 Church Street, Ann Arbor, MI 48109-1040, USA \\ Raman Research Institute \\ C V Raman Avenue, Sadashivanagar, Bangalore, 560 080, India \\ E-mail: romanerri.res.in

\section{Luca Bombelli} \\ Department of Physics and Astronomy, University of Mississippi \\ 108 Lewis Hall, University, MS 38677-1848, USA \\ Departament de Física Fonamental, Universitat de Barcelona \\ Av. Diagonal 647, 08028 Barcelona, Spain \\ E-mail: luca@phy.olemis. .edu
}

\begin{abstract}
We describe a framework for constructing Lagrangians for fields propagating on the background of a locally finite partially ordered set where the partial order corresponds to causal relations, a causal set. We require that, when the causal set is well approximated by a spacetime manifold, the Lagrangians agree with the usual continuum ones, so most of our procedure consists in coming up with expressions for the latter that are meaningful when the structure available is that of a causal set. Given a physical field, the basic step is the definition of one or more "Lagrangian generators" for the field, each of which depends on the field and on the choice of a small set of points in the causal set. With these, one then finds quasilocal "pre-Lagrangians" that depend on Alexandrov sets, regions causally located between two points $p \prec q$, and finally local Lagrangian densities. We describe in detail the case of scalar and electromagnetic fields.
\end{abstract}

Workshop on Continuum and Lattice Approaches to Quantum Gravity 17-19 September 2008

University of Sussex, United Kingdom

\footnotetext{
*Speaker.
} 


\section{Introduction}

It is well known that a standard way to do quantum gravity is to discretize spacetime. Ironically, this discretization violates the very principles of relativity the theory, at least in its usual approaches, tries to preserve. For example, if a spacetime is viewed as having a cubic lattice structure, the edges of the lattice single out "preferred directions". For that reason, of course, most approaches to quantum gravity do not use cubic lattices, or any other structure recognizable on a large scale. However, there are still bothersome violations of relativity on a small scale in these theories (even though the problem might be cured after path integration).

One purpose of causal set theory is to allow discretization and relativity to peacefully co-exist. This is done by viewing spacetime as a (locally) finite partially ordered set [1] (for more recent reviews, see references $[2,3,4])$. The partial order, interpreted as the causal relations determined by the lightcone, is, by definition, covariant. In the case of a continuum Lorentzian manifold $\mathscr{M}$, that partial order, $\prec \mathscr{M}$, is defined as follows: $p \prec \mathscr{M} q$ if and only if one can travel from $p$ to $q$ without going faster than the speed of light. In terms of the spacetime metric $g_{\mu v}$, if the lightcone at a point $x \in \mathscr{M}$ is fixed, the only freedom left is that of performing a conformal transformation, which modifies the volume element $\sqrt{|\operatorname{det} g|}$. The global version of this statement is the result by Hawking et al [5] and Malament [6] that for reasonable spacetimes (e.g., without closed timelike curves) there is a bijection between causal relations $\prec \mathscr{M}$ and conformal structures $g_{\mu v} /|\operatorname{det} g|^{1 / d}$. In a discrete scenario, the role of the volume element is played by a simple count of points, which suggests that $\prec$ can replace $g_{\mu \nu}$.

However, the set of all locally finite partial orders $(S, \prec)$ is much larger than the set of discrete manifoldlike ones. ${ }^{1}$ Thus, the replacement $g_{\mu \nu} \mapsto \prec$ introduces new degrees of freedom, and $\prec-$ based gravity is no longer equivalent to the $g_{\mu \nu}$-based theory of gravity we are familiar with. ${ }^{2}$ This, however, leads to a natural approach to modeling the breakdown of the manifold structure at small scales due to quantum fluctuations. One burden for such a theory is to invent a dynamics that explains why at large scales $\prec \approx \prec \mathscr{M}$, for some manifold $\mathscr{M}$. This is still an unresolved issue that is beyond the scope of this talk.

Instead, our goal here is to formulate a theory describing a quantum field propagating on a causal set. Let $\mathscr{F}_{\mathscr{M}}$ be a set of fields of interest on a manifold $\mathscr{M}$, with tensor fields of different types defined in the usual way. Let $\mathscr{F}_{S}$ be the corresponding set of fields on an arbitrary causal set $S$; while the definition of a scalar field on $S$ is straightforward, other types of fields must be defined in a radically different way that does not rely on the presence of a manifold structure. Given a "sprinkling" map $f: S \rightarrow \mathscr{M}$, for any field $F_{\mathscr{M}} \in \mathscr{F}_{\mathscr{M}}$ on $\mathscr{M}$ one can define its pullback on $S$, $F_{S}=f^{*} F_{\mathscr{M}}$. The goal of the theory is to define a Lagrangian density $\mathscr{L}_{S}: \mathscr{F}_{S} \times S \rightarrow \mathbb{R}$ in such a way that the following two conditions are met: (1) In the case of a manifoldlike causal set $S$ that is close to a manifold $\mathscr{M}$, and a field on $S$ that can be considered as the pullback of a field on $\mathscr{M}$,

\footnotetext{
${ }^{1} \mathrm{~A}$ manifoldlike causal set $S$ is one such that there is a Lorentzian manifold $(\mathscr{M}, g)$ and an order-preserving mapping $f: S \rightarrow \mathscr{M}$ under which $f(S)$ appears as a set of points distributed with uniform density $\rho$ in $\mathscr{M}$, and the average point separation is much smaller than any scale set by the geometry or topology of the manifold; thus, $S$ can be seen as a discretization of $\mathscr{M}$.

${ }^{2}$ Notice that there is one sense in which this theory is more restrictive than general relativity. Since the basic structure is a partial order, no manifolds with "timelike loops" can arise in the large-scale, continuum approximation.
} 
$\mathscr{L}_{S}\left(f^{*} F, x\right) \approx \mathscr{L}_{\mathscr{M}}\left(F_{\mathscr{M}}, f(x)\right)$; and (2) $\mathscr{L}_{S}\left(F_{S}, x\right)$ is well defined when the causal set and fields are non-manifoldlike, and the fields cannot be construed as pullbacks.

This goal can be accomplished by rewriting field theory Lagrangians in a way that is amenable to causal set discretization. In other words, it should rely on timelike distances, volumes, and causal relations. Both of the latter are well defined for a causal set: the volume of $T \subset S$ is defined to be the number of elements in $T$ multiplied by some constant, while the distance $\tau_{S}$ between causally related pairs of points $p \prec q$ is defined as the length of the longest possible chain $p \prec r_{1} \prec \ldots \prec$ $r_{n} \prec q$. The pullback of $\prec_{g, \mathscr{M}}$ under $f: S \rightarrow \mathscr{M}$ is a causal relation $\prec_{f g}$ on $S$ in which $p \prec_{f g} q$ if and only if $f(p) \prec_{g, \mathscr{M}} f(q)$. It is known that if the measure $\mu_{\mathscr{M}}$ and Lorentzian distance $\tau_{\mathscr{M}}$ on a manifold $\mathscr{M}$ are defined in the usual, continuum-based way, then $\mu_{S}\left(f^{-1}(\mathscr{N})\right) \approx \rho \mu_{\mathscr{M}}(\mathscr{N})$, from properties of uniform point distributions, and $\tau_{S}(p, q) \approx \kappa_{d}^{-1} \tau_{\mathscr{M}}(f(p), f(q))$, where $\kappa_{d}$ is a dimension-dependent coefficient [7]. At the same time, however, both volumes and distances are defined for non-manifoldlike causal sets as well.

The goal of this talk is to construct Lagrangians for two types of fields defined on causal sets, scalar and Abelian gauge fields. On a manifold, they are defined respectively as functions $\phi: \mathscr{M} \rightarrow \mathbb{R}$ and connection forms $A: \mathscr{M} \rightarrow \mathrm{T}^{*} \mathscr{M}$. In a causal set, on the other hand, they are defined as $\phi: S \rightarrow \mathbb{R}$ and $a: S \times S \rightarrow \mathbb{R}$, respectively. The pullback of a scalar field is defined as $\left(f^{*} \phi\right)(x)=\phi(f(x))$, while the pullback of a one-form, $\left(f^{*} A\right)(p, q)$, is a line integral of $V^{\mu}$ along the geodesic connecting $p$ and $q$ (to avoid unnecessary complications, we will assume that the geodesic is unique). Our task is to define $\mathscr{L}_{S}$ in such a way that $\mathscr{L}_{S}\left(f^{*} \phi, x\right) \approx \mathscr{L}_{\mathscr{M}}(\phi, f(x))$ and $\mathscr{L}_{S}\left(f^{*} A, x\right) \approx \mathscr{L}_{\mathscr{M}}(A, f(x))$. Gravitational and fermionic Lagrangians are beyond the scope of the talk, but they have been adressed in references [8, 9, 10] and references [11, 10], respectively, and non-Abelian gauge fields can be treated with an extension of the formalism described here.

\section{Lagrangians, pre-Lagrangians and Lagrangian generators}

In the case of discretized Riemannian manifolds, the continuum Lagrangian density $\mathscr{L}(F ; x)$ for a field $F$ can be replaced by a "pre-Lagrangian" $\tilde{\mathscr{L}}\left(F ; n_{\rho}(x)\right)$ that depends on the value of $F$ at a small number of nearest neighbors, points contained in the ball $n_{\rho}(x)$ of radius $\rho$ around $x$ (for a gravity-related example see, e.g., reference [12]). In the Lorentzian case, however, $n_{\rho}(x)$ may not be compact and may contain infinitely many points. Therefore, we replace it by an Alexandrov set or interval between two causally related points,

$$
\alpha(p, q)=\{r \mid p \prec r \prec q\},
$$

which includes $x$ among its elements. Such an Alexandrov set is clearly compact in a Lorentzian manifold $\mathscr{M}$, and it is finite in the case of a general causal set, because of its local finiteness.

The issue we need to address is the fact that, contrary to the Riemannian $n_{\rho}(x)$, the Alexandrov set $\alpha(p, q)$ is not unique, even if we specify its height $\tau_{0}$, and its choice will in general affect the value of $\tilde{\mathscr{L}}(F ; \alpha(p, q))$. Physically, a choice of $\alpha(p, q)$ corresponds to a reference frame, in which the time direction is along the geodesic from $p$ to $q$. In the case of a differentiable field in a continuum, this violation of relativity can be avoided by defining the time direction of the preferred frame, $v^{\mu}$, by a covariant expression involving $F$. For example, for scalar field $F=\phi$, we could pick $v^{\mu}=\partial^{\mu} \phi$, which turns a non-covariant expression such as $\mathscr{L}=v^{\mu} \partial_{\mu} \phi$ into a covariant one, 
$\mathscr{L}=\partial^{\mu} \phi \partial_{\mu} \phi$. In a causal set $S$, we can use the dynamics of a field $F_{S}$ to select a collection $\beta\left(F_{S}, x\right)$ of preferred Alexandrov sets containing $x$. In a generic case, $\beta\left(F_{S}, x\right)$ consists of a single element, which corresponds to single preferred frame; but in general this doesn't have to be the case.

The dynamics is determined by a set of "Lagrangian generators" $\mathscr{K}: D \times \mathscr{F}_{S} \rightarrow \mathbb{R}$, where $D$ is a subset of $S^{n}$ for some $n$; in other words, $\mathscr{K}$ is a function of an $n$-tuple of causal set points and a field. For each Lagrangian generator $\mathscr{K}$, the preferred Alexandrov sets $\beta\left(F_{S}, x\right)$ around $x \in S$ are those that minimize the variation

$$
\Delta \mathscr{K}=\max \left\{\mathscr{K}\left(T, F_{S}\right) \mid T \in D \cap \alpha(p, q)^{i}\right\}-\min \left\{\mathscr{K}\left(T, F_{S}\right) \mid T \in D \cap \alpha(p, q)^{i}\right\}
$$

of $\mathscr{K}$ in their interior, with the constraint that $\tau_{S}(p, q)=\tau_{0}$. In this expression the maximum and minimum are over $T$, which means that $\Delta \mathscr{K}$ is a function of $F_{S}$ and $\alpha(p, q)$ alone, and generically we expect it to be minimized by a single $\alpha$, thus turning $\beta\left(F_{S}, x\right)$ into a one element set.

Given a Lagrangian generator $\mathscr{K}$, the pre-Lagrangian corresponding to an Alexandrov set $\alpha(p, q)$ is defined as the average of the largest and smallest values of $\mathscr{K}$ in its interior:

$$
\begin{aligned}
& \mathscr{L}\left(F_{S}, \alpha(p, q)\right) \\
& =\frac{1}{2}\left(\max \left\{\mathscr{K}\left(T, F_{S}\right) \mid T \in D \cap \alpha(p, q)^{i}\right\}+\min \left\{\mathscr{K}\left(T, F_{S}\right) \mid T \in D \cap \alpha(p, q)^{i}\right\}\right) .
\end{aligned}
$$

Finally, we define the Lagrangian density $\mathscr{L}_{S}\left(F_{S}, x\right)$ as the average of the densities corresponding to each candidate Alexandrov set around $x$,

$$
\mathscr{L}\left(F_{S}, x\right)=\frac{\sum_{\alpha \in \beta\left(F_{S}, x\right)} \tilde{\mathscr{L}}\left(F_{S}, \alpha\right)}{\sharp\left\{\beta\left(F_{S}, x\right)\right\}} .
$$

Notice that, as a consequence of the procedure for obtaining $\mathscr{L}$ from $\mathscr{K}$, only $\mathscr{L}$ is additive, while $\mathscr{K}$ is not. If we need several Lagrangian generators to define a Lagrangian density, because a field requires more than one generator and/or because there are two or more fields, we write

$$
\mathscr{K}=\left\{\mathscr{K}_{1}, \ldots, \mathscr{K}_{n}\right\} ; \quad \mathscr{L}(\mathscr{K})=\sum_{i=1}^{n} \mathscr{L}\left(\mathscr{K}_{i}\right) .
$$

\section{Scalar field Lagrangian}

Assuming that there is no interaction with the electromagnetic field (this corresponds to setting $a(r, s)=0$ in the next section), a massive scalar field $\phi$ has a set of three Lagrangian generators,

$$
\mathscr{K}_{\text {scal }}=\left\{\mathscr{K}_{\mathrm{t}}, \mathscr{K}_{\mathrm{s}}, \mathscr{K}_{\mathrm{m}}\right\},
$$

where the "timelike", "spacelike" and "mass" Lagrangian generators are, respectively,

$$
\begin{aligned}
& \mathscr{K}_{\mathrm{t}}\left(r_{\mathrm{t}}, s_{\mathrm{t}} ; \phi\right)=c_{\mathrm{t}}\left(\phi\left(s_{\mathrm{t}}\right)-\phi\left(r_{\mathrm{t}}\right)\right)^{2} \\
& \mathscr{K}_{\mathrm{s}}\left(r_{\mathrm{s}}, s_{\mathrm{s}} ; \phi\right)=-c_{\mathrm{s}}\left(\phi\left(r_{\mathrm{s}}\right)-\phi\left(s_{\mathrm{s}}\right)\right)^{2} \\
& \mathscr{K}_{\mathrm{m}}(r, s, \phi)=\frac{1}{4} m^{2}\left(r \prec^{?} s\right)(\phi(r)+\phi(s))^{2} .
\end{aligned}
$$

Here, $\prec^{?}$ is defined in such a way that $a \prec^{?} b$ is 1 if the relation $a \prec b$ holds, and 0 otherwise. The domains corresponding to $\mathscr{K}_{\mathrm{m}}, \mathscr{K}_{\mathrm{t}}$ and $\mathscr{K}_{\mathrm{s}}$ are $D_{\mathrm{m}}, D_{\mathrm{t}}$ and $D_{\mathrm{s}}$ respectively, defined as

$$
\begin{aligned}
& D_{\mathrm{m}}=S \times S, \quad D_{\mathrm{t}}=\{(p, q) \mid p \prec q\}, \\
& D_{\mathrm{s}}=S \times S \backslash\{(p, q) \mid p \prec q\} \backslash\{(p, q) \mid q \prec p\} .
\end{aligned}
$$


The mass term of the above Lagrangian density is especially easy to compute. Assume that $\phi$ is constant, $\phi(x)=c$ for all $x$, and that every Alexandrov set $\alpha(p, q)$, with $\tau(p, q)=\tau_{0}$ contains at least one spacelike-separated pair of points. In this case, all such Alexandrov sets have $\Delta \mathscr{K}_{\mathrm{m}}=m^{2} \phi^{2}$, and thus they are all elements of $\beta(\phi ; x)$. They all have identical pre-Lagrangians, which are equal to $\frac{1}{2} m^{2} \phi^{2}$, which means that the value of the actual Lagrangian density is the same.

In order to compute the kinetic part of the Lagrangian density, consider the example of a pullback $f^{*} \phi$, where $f: S \rightarrow \mathscr{M}$ is the result of a random Poisson point process and $\phi: \mathscr{M} \rightarrow \mathbb{R}$ is linear. Then this problem can be split into four parts:

(1) $\mathscr{K}=\mathscr{K}_{\mathrm{t}}$ when the gradient of $\phi$ is timelike,

(2) $\mathscr{K}=\mathscr{K}_{\mathrm{t}}$ when the gradient of $\phi$ is spacelike,

(3) $\mathscr{K}=\mathscr{K}_{\mathrm{s}}$ when the gradient of $\phi$ is timelike,

(4) $\mathscr{K}=\mathscr{K}_{\mathrm{s}}$ when the gradient of $\phi$ is spacelike.

If $\alpha(p, q)$ minimizes the variation of $f^{*} \phi$ under the constraint $\tau_{S}(p, q)=\tau_{0}$, then $\alpha(f(p), f(q))$ approximately corresponds to the minimum of the variation of $\phi$ under the constraint $\tau_{\mathscr{M}}(u, v) \approx \tau_{0}$. It can be easily seen that, in order for the latter to happen, the direction of the gradient of $\phi$ should approximately coincide with the axis of $\alpha(f(p), f(q))$ in cases 1 and 3, and it should approximately lie on the equator of $\alpha(f(p), f(q))$ in cases 2 and 4 . In all four cases, we can select a coordinate system in such a way that $p=(-\tau / 2,0,0,0)$ and $q=(\tau / 2,0,0,0)$. In cases 2 and 4 we can also select the $x$ axis to be parallel to the gradient of $\phi$. Thus, if $\phi$ is linear, then $\phi=k t$ in cases 1 and 3 , and $\phi=k x$ in cases 2 and 4 .

The maximal variation of $\phi$ is represented by points $r$ and $s$, and in all cases $f(r) \approx-f(s)$. In case 1 , it is easy to see that $r=p$ and $s=q$. In case $4, f(r)=(0,-\tau / 2,0,0)$, and $f(s)=$ $(0, \tau / 2,0,0)$. However, in cases 2 and 3, the choices of $r$ and $s$ are limited by the fact that $r$ and $s$ are supposed to be respectively timelike-separated and spacelike-separated, which, in both cases, forces us to choose them in such a way that $f(r) \approx(-\tau / 4,-\tau / 4,0,0)$ and $f(s) \approx(\tau / 4, \tau / 4,0,0)$.

By combining all four cases, the total Lagrangian density based on $\left\{\mathscr{K}_{\mathrm{t}}, \mathscr{K}_{\mathrm{s}}\right\}$ is given by

$$
\mathscr{L}= \begin{cases}\left(d_{\mathrm{t}}+\frac{1}{4} d_{\mathrm{s}}\right) \partial^{\mu} \phi \partial_{\mu} \phi & \text { if } \quad \partial^{\mu} \phi \partial_{\mu} \phi>0 \\ -\left(d_{\mathrm{s}}+\frac{1}{4} d_{\mathrm{t}}\right) \partial^{\mu} \phi \partial_{\mu} \phi & \text { if } \quad \partial^{\mu} \phi \partial_{\mu} \phi<0 .\end{cases}
$$

Since whether or not $\partial^{\mu} \phi \partial_{\mu} \phi$ is greater than 0 is relativistically covariant, the above definition of the Lagrangian density is relativistically covariant as well. Thus, in principle, there is nothing wrong with the above Lagrangian density, except for the fact that, of course, it does not in general coincide with the usual Klein-Gordon Lagrangian density. The latter, however, is a special case of the one we obtained above for $c_{\mathrm{s}}=-c_{\mathrm{t}}$ or, equivalently, $d_{\mathrm{s}}=-d_{\mathrm{t}}$. This gives

$$
\mathscr{L}=\frac{3}{4} d_{\mathrm{t}} \partial^{\mu} \phi \partial_{\mu} \phi
$$

\section{Lagrangian for Abelian gauge fields}

In a manifold $\mathscr{M}$, the basic variable for the electromagnetic field is the vector potential or connection 1-form $A_{\mu}$. Its causal-set counterpart corresponds to an integrated form of the connection, 
an object of the type $a: S \times S \rightarrow \mathbb{R}$. If $f: S \rightarrow \mathscr{M}$ is a sprinkling on $\mathscr{M}$, we will say that $a$ is the pullback of $A\left(a=f^{*} A\right)$, if

$$
a(p, q)=\int_{\gamma(f(p), f(q))} g_{\mu v} A^{\mu} \mathrm{d} x^{v}
$$

whenever $f(p)$ and $f(q)$ are connected by a unique geodesic $\gamma(f(p), f(q))$.

Before introducing the Lagrangian generators, we will define their domain $D$. While in future work it might be interesting to investigate more general forms for the domain, for present purposes we select the simplest one,

$$
D=\left\{\left(r_{1}, r_{2}, s_{1}, s_{2}, s_{3}, s_{4}\right) \mid \tau\left(r_{1}, r_{2}\right)=\tau_{0} ; r_{1} \prec s_{k} \prec r_{2} ; \tau\left(r_{1}, s_{k}\right)+\tau\left(s_{k}, r_{2}\right)<\varepsilon\right\},
$$

where $\varepsilon$ is some small number. The constraint $\tau\left(r_{1}, r_{2}\right)=\tau_{0}$ implies that when going from the Lagrangian generator to the Lagrangian density, the points $r_{1}$ and $r_{2}$ will be forced to coincide with $p$ and $q$ respectively. The second constraint, $\tau\left(r_{1}, s_{k}\right)+\tau\left(s_{k}, r_{2}\right)<\varepsilon$, is equivalent to saying that points $f\left(s_{1}\right)$ through $f\left(s_{4}\right)$ lie very close to the boundary of the equator of the Alexandrov set.

There will be two Lagrangian generators, the "timelike" and "spacelike" one, respectively:

$$
\begin{aligned}
& \mathscr{K}_{\mathrm{t}}\left(r_{1}, r_{2}, s_{1}, s_{2}, s_{3}, s_{4}\right)=\left(a\left(r_{1}, s_{1}\right)+a\left(s_{1}, r_{2}\right)+a\left(r_{2}, s_{2}\right)+a\left(s_{2}, r_{1}\right)\right)^{2} \\
& \mathscr{K}_{\mathrm{s}}\left(r_{1}, r_{2}, s_{1}, s_{2}, s_{3}, s_{4}\right)=\left(a\left(s_{1}, s_{2}\right)+a\left(s_{2}, s_{3}\right)+a\left(s_{3}, s_{4}\right)+a\left(s_{4}, s_{1}\right)\right)^{2} .
\end{aligned}
$$

Now we notice that, for the special case of $a=f^{*} A$,

$$
\begin{aligned}
& \left(f^{*} A\right)(t, u)+\left(f^{*} A\right)(u, v)+\left(f^{*} A\right)(v, w)+\left(f^{*} A\right)(w, t) \\
& =\int_{\gamma(f(t), f(u))} A_{\mu} \mathrm{d} x^{\mu}+\int_{\gamma(f(u), f(v))} A_{\mu} \mathrm{d} x^{\mu} \\
& \quad+\int_{\gamma(f(v), f(w))} A_{\mu} \mathrm{d} x^{\mu}+\int_{\gamma(f(w), f(t))} A_{\mu} \mathrm{d} x^{\mu}=\int_{\text {closed contour }} A_{\mu} \mathrm{d} x^{\mu} .
\end{aligned}
$$

Consider a coordinate system in which $f(p)=(-\tau / 2,0,0,0)$ and $f(q)=(\tau / 2,0,0,0)$. By noticing their correspondence to fluxes through these square loops whose edges are four geodesic segments, it is easy to see that $\mathscr{K}_{\mathrm{s}}$ and $\mathscr{K}_{\mathrm{t}}$ vary from 0 to $|\vec{B}|^{2} \tau^{2}$ and $|\vec{E}|^{2} \tau^{2}$ respectively.

It can be shown that $|\vec{B}|^{2} \tau^{2}$ and $|\vec{E}|^{2} \tau^{2}$ are both minimized in the reference frame in which $\vec{E}$ and $\vec{B}$ are parallel, and the existence of that frame can be easily demonstrated. The specific values of $|\vec{B}|^{2} \tau^{2}$ and $|\vec{E}|^{2} \tau^{2}$ in that reference frame however can be expressed covariantly as scalars representing solutions to the following system of equations:

$$
E^{2}-B^{2}=F^{\mu v} F_{\mu v} ; \quad E B=\frac{1}{8} \varepsilon_{\alpha \beta \gamma \delta} F^{\alpha \beta} F^{\gamma \delta} .
$$

These solutions are

$$
E^{2}=\frac{1}{2}\left[F^{\mu v} F_{\mu v}+\sqrt{\left(F^{\mu v} F_{\mu v}\right)^{2}+\frac{4}{k^{2}}\left(\varepsilon_{\alpha \beta \gamma \delta} F^{\alpha \beta} F^{\gamma \delta}\right)^{2}}\right]
$$

and

$$
B^{2}=\frac{1}{2}\left[\sqrt{\left(F^{\mu v} F_{\mu v}\right)^{2}+\frac{4}{k^{2}}\left(\varepsilon_{\alpha \beta \gamma \delta} F^{\alpha \beta} F^{\gamma \delta}\right)^{2}}-F^{\mu v} F_{\mu v}\right] .
$$

The Lagrangian generators for these are respectively the $\mathscr{K}_{\mathrm{t}}$ and $\mathscr{K}_{\mathrm{s}}$ defined earlier. Both produce Lorentz-covariant expressions; they simply include a Lorentz contraction that does not exist in practice. This can be easily fixed by setting

$$
\mathscr{K}=\left\{\mathscr{K}_{\mathrm{t}},-\mathscr{K}_{\mathrm{s}}\right\} .
$$




\section{Getting Rid of Unwanted Fluctuations}

Throughout this work one basic assumption was made: the dynamical fields depend linearly on the coordinates in the region of interest. However, given a generic slowly-varying field $\phi$, that assumption will only be valid in a set of preferred frames of finite measure near the "center of mass frame" of the perturbation, and not in the vicinity of the light cone, which has infinite measure.

Physically, this means that if two objects move with arbitrary velocity relative to each other, then that velocity is arbitrarily close to the speed of light, so the objects look like $\delta$-function matter distributions to each other and do not resemble locally linear fields. Therefore the approximations that were used in this paper, which depend on approximate linearity, are less likely to be good when there are large relative speeds. This is one of the secondary reasons why the distance-based topology has been replaced by the Alexandrov topology, since, in the case of differentiable functions, approximate linearity does locally hold in the latter case. Since the procedure of selecting the Alexandrov sets in $\beta(F, x)$ does not differentiate between linear and non-linear variations, it is likely to select Alexandrov sets where the fluctuations of the fields are not too high. This procedure does not violate relativity because the preferred frame is covariantly defined, based only on the behavior of the fields. A good analogy to think about is the Earth acting as a preferred frame in geophysics.

\section{Conclusion}

In this work we showed how to introduce Lagrangian densities for two types of bosonic fields on a general partially ordered set. Furthermore, we showed that those Lagrangians densities approximate well the ones we are familiar with from manifold-based quantum field theory when the causal set is manifoldlike (i.e., in the special case when both causal structure and fields are defined as pullbacks from a manifold). While the Lagrangian densities themselves might look complicated, it turns out that they can be "read off" from suitable Lagrangian generators. Thus, in this theory, (sets of) Lagrangian generators replace Lagrangian densities as the defining objects of a theory.

However, while Lagrangians are defined, what to do with them is an open question. As we all know, the fields at every point in spacetime are viewed as separate degrees of freedom in path integration. In regular quantum field theory one can imagine that spacetime looks like a cubic lattice, where the set of "nearest neighbors" of each point is predetermined and not field-dependent, which greatly simplifies the path integral. In causal set theory, however, there is no such regular structure; instead, the Alexandrov sets to be used in the pre-Lagrangian are field-dependent, and must be determined on a case-by-case basis.

One way to make oneself feel better about this situation is to point out that even Einstein's equation has very few analytically known exact solutions. However, in order to be able to bring this theory to the level of general relativity there have to be at least some special cases that can be solved in it. One idea that comes to mind, of course, is a causal set consisting of very few points. However, unlike the situation with exact solutions to Einstein's equation, this situation is not physical. It is important to search for many-point universes that can be treated exactly, and to understand how large-scale manifoldlike structures arise dynamically. These are still subjects for future research, but for some ideas on possible ways to address the latter issue, see reference [10]. 


\section{References}

[1] L. Bombelli, J. Lee, D. Meyer and R.D. Sorkin, Space-time as a causal set, Phys. Rev. Lett. 59 (1987) 521-524.

[2] R.D. Sorkin, Causal sets: discrete gravity, in Lectures on Quantum Gravity, eds A. Gomberoff and D. Marolf (New York: Springer 2005) [gr-qc/0309009].

[3] F. Dowker, Causal sets and the deep structure of spacetime, in 100 Years of Relativity - Space-time Structure: Einstein and Beyond, ed A. Ashtekar, World Scientific 2005 [gr-qc/0508109].

[4] J. Henson, The causal set approach to quantum gravity, in Approaches to Quantum Gravity: Toward a New Understanding of Space, Time and Matter, ed D. Oriti, Cambridge University Press 2009 [gr-qc/0601121].

[5] S.W. Hawking, A.R. King and P.J. McCarthy, A new topology for curved spacetime which incorporates the causal, differential and conformal structures, J. Math. Phys. 17 (1976) 174-181.

[6] D. Malament, The class of continuous timelike curves determines the topology of spacetime, J. Math. Phys. 18 (1977) 1399-1404.

[7] G. Brightwell and R. Gregory, Structure of random discrete spacetime, Phys. Rev. Lett. 66 (1991) $260-263$.

[8] R. Sverdlov and L. Bombelli, Gravity and matter in causal set theory, Class. Quantum Grav. 26 (2009) 07511 [arXiv:0801.0240].

[9] R. Sverdlov and L. Bombelli, Dynamics for causal sets with matter fields: A Lagrangian-based approach, contribution to the Proceedings of the DICE2008 Conference, J. Phys.: Conf. Ser. 174 (2009) 012019 [arXiv:0905.1506].

[10] R. Sverdlov, Quantum Field Theory and Gravity in Causal Sets, University of Michigan Ph.D. dissertation, arXiv:0905.2263.

[11] R. Sverdlov, Spinor fields in causal set theory. arXiv:0808.2956.

[12] L. Bombelli, A. Corichi and O. Winkler, Semiclassical quantum gravity: Statistics of combinatorial Riemannian geometries, Annalen der Physik 14 (2005) 499-519 [gr-qc/ 0409006 ]. 